编者按.

Wallace (Wally) S. Broecker 1958 年获得哥伦比亚大学博士学位, 1976 年当选为美国艺术与科学学院院士, 1979 年当 选为美国科学院院士. 目前是纽约哥伦比亚大学 Lamont-Doherty 地球观测中心地球与环境科学领域教授, 同时是多个著 名学会的特别会员. Wally 因在地球和环境科学领域所做出的杰出贡献而荣获诸多科学奖章和奖励, 如 1979 年和 1995 年 分别获得美国地球物理学会的 Maurice Ewing 奖章和 Roger Revelle 奖章; 1984 年和 2000 年分别获得美国地质学会的 Arthur Day 奖章和 Don Easterbrook 杰出科学家奖; 1986 年获得欧洲地球物理学会 Urey Medal 奖章, 地球化学学会 Goldschmidt 奖章; 1986 年获得美国科学院 Alexander Agassiz 奖章; 1990 年获得伦敦地质学会 Wollaston 奖章; 1996 年获得 美国国家科学奖章, 东京 Asahi 格拉斯基金会 Blue Planet 奖; 2002 年获得美国科学院 Arthur Day 奖, 同年和刘东生院士一 起获得国际 Tyler 环境科学成就奖.

Wally 在国际地球生物学、地球化学和地球物理学研究领域享有盛名. 他的研究兴趣包括古气候学、海洋化学、同 位素地球化学、地质年代学和环境科学等. Wally 尤以对海洋环流中大输送带的深刻认识而著名于全世界, 这条输送带在 全球气候中发挥着关键的作用(The Great Ocean Conveyor. Oceanography, 1991, 4: 79 - 89). 他指出: “气候系统是一头发怒 的野兽, 而我们正在攻击它并激其发怒”; “当地球系统从一种模式转换到另一种模式时, 全球气温将发生剧烈的波动”; “由 温室效应引起的适度大气升温也可能导致相似的全球气温的剧烈变化”. 事实上, Wally 在国际上不仅强调“地球气候将受 到大气温室气体浓度增加的潜在威胁”, 而且还率先提出了一系列全球气候系统的研究方法, 包括应用 C 和其他同位素对 海底沉积物测年的方法. 他确立了随时间变化的海洋环流模式, 探索海洋与大气之间的气体交换, 追踪 C 元素在地球化 学、物理和生物系统间的循环过程. Wally 发表研究论文数百篇, 出版近 10 部著作. 他还是一位出色的导师, 培养的学生 中今天不少已成为地球与环境科学及相关领域的国际领衔科学家.

我不是一位环境科学家, 但对这一领域有浓厚的兴趣. 这一兴趣来自于 Wally 的研究生和博士后对我的深刻启发和 影响, 当时我在 Lamont 做博士后(1992 1993 年), 曾和他们一起共用办公室和实验设备. 也许, 在这段时间里我听到的 “全球变暖”、“气候变化”、“冰盖”、“冰芯”、“海洋环流”等比我所研究的“洋脊玄武岩”要多得多. 今天, 在我的家乡所 在的中国西北农村, 孩子们似乎已不能像我年少时一样堆雪人、在河上溜冰了. 这使我相信, 全球逐渐变暖似乎已成为事 实. 但究竟是什么因素导致了全球变暖的发生, 人类活动释放的温室气体对此贡献有多大, 依然争议不定. 然而, 一个不 争的事实是, 如果全球变暖继续下去, 冰盖将会被融化, 我们将面临严重的后果. 中国经济和个人财富空前的增长使我 深受鼓舞并使我自豪, 但每天增加的大量汽车将消耗越来越多的化石燃料, 释放越来越多的温室气体, 这对我们生活环 境中的空气产生直接的污染和影响. 但如何评价这样一个世界人口最多, 并在未来 10 年或者 20 年拥有巨大汽车发展潜 力的国家对全球变暖的贡献呢? Wally 在该研究领域有他独特的观点和见解. 我非常高兴 Wally 欣然接受邀请为《科学通 报》撰写该文, 阐述自己的论点.

(牛耀龄，执行副主编, Department of Earth Sciences, Durham University, UK)

\title{
全球变暖: 行动还是等待?
}

W. S. Broecker

(Lamont-Doherty Earth Observatory of Columbia University, 61 Route 9W, PO Box 1000, Palisades, NY 10964-8000, USA. E-mail: broecker@ldeo.columbia.edu)

摘要 人类怎样应对大气 $\mathrm{CO}_{2}$ 浓度的持续升高, 仍有较大的分歧. 一部分人认为, 除非全球升温更趋显 著, 否则各项耗资巨大的措施应推延执行; 而另一部分人则认为, 即便是马上采取措施, 大气中 $\mathrm{CO}_{2}$ 浓 度也可能已达到无法接受的水平, 因而必须即刻采取各种措施. 我本人属于后者. $\mathrm{CO}_{2}$ 的有些影响已经 达到地球轨道周期性变化、海洋环流重新组合或太阳辐射变化所导致的效应. 这些效应用模型无法揭示 出来, 而在古气候记录里被清楚地保存下来. 这使我觉得这些模型缺乏自然界客观存在的反馈和放大 效应, 因而很可能低估了 $\mathrm{CO}_{2}$ 浓度升高的影响, 而不是高估. 世界能源消费将持续增长, 煤炭由于其廉 价性和丰富的蕴藏将成为未来主要的能源. 我同时认为, $\mathrm{CO}_{2}$ 的固定和储藏在阻止大气 $\mathrm{CO}_{2}$ 浓度的升高 方面有关键作用. 所幸这些固定和储藏在技术和经济层面上都是可行的. 目前面临的一个重大问题是, 在 $\mathrm{CO}_{2}$ 浓度达到自然界无法承受的水平之前, 全球能否一起合作, 使减缓措施尽快付诸行动.

关键词 全球变暖 $\mathrm{CO}_{2}$ 吸存 古气候 
在人类活动对自然界的影响中, 大气中逐渐升 高的温室气体 $\left(\mathrm{CO}_{2}, \mathrm{CH}_{4}, \mathrm{~N}_{2} \mathrm{O}\right)$ 浓度已成为最具争议 性的话题. 环境保护论者认为, 如果目前不采取有力 措施, 在末来的一个世纪, 大气中温室气体浓度的持 续增加导致的全球升温将对全球农业生产、淡水供应 和野生动植物资源产生严重的影响. 两极地区冰盖 的融化也将导致海平面上升, 使宝贵的海岸资源遭 到严重破坏. 目前, 高山冰川不断融化、北极圈海冰 范围逐渐减少以及冻原土壤解冻等现象已经表明全 球升温的影响已经在进行. 这些自然变化提示我们, 为了避免全球升温更大的影响, 人类必须阻止煤炭、 石油和天然气燃烧产生的 $\mathrm{CO}_{2}$ 在大气中的累积.

尽管几乎所有的科学家都认识到全球升温产生 的诸多负面影响, 相当数量的公众却不以为然. 美 国、澳大利亚、中国、欧洲以及日本达成协议以削减 $\mathrm{CO}_{2}$ 排放, 但这些国家中部分政府的沉默寊言就能反 应出这种不以为然的态度. 他们认为, 化石燃料的正 常商业消耗所产生的长远结果是不确定的; 相比之 下, $\mathrm{CO}_{2}$ 减排导致的经济后果过于沉重.

\section{$1 \mathrm{CO}_{2}$ 排放}

控制排放所面临的艰巨性使形势更趋复杂. 2005 年, 全球化石燃料燃烧排放 $\mathrm{CO}_{2}$ 约 250 亿吨. 这样数 量的 $\mathrm{CO}_{2}$ 如果被固定液化, 将填充满一个边长和深度 各为 $3 \mathrm{~km}$ 体积的容器. 如果排除森林采伐对 $\mathrm{CO}_{2}$ 排 放的贡献, 自工业革命以来大气中 $\mathrm{CO}_{2}$ 增加的 $30 \%$ 将 归结于化石燃料燃烧. 如果煤炭、石油和天然气燃烧 排放的 $\mathrm{CO}_{2}$ 全部驻留在大气中, 大气增加的 $\mathrm{CO}_{2}$ 将是 目前的两倍, 这一点是无可非议的. 很多研究表明, 由化石燃料燃烧排放 $\mathrm{CO}_{2}$ 的另外一半, 大部分被海洋 所吸收 ${ }^{[1]}$, 剩余的少部分储存在植被和土壤中. 囊括 大气、海洋和陆地生物圈的三箱模型表明, 大气和海 洋(包括陆地生物圈)各储存 $50 \% \mathrm{CO}_{2}$ 的模式将贯穿 末来的一个世纪.

目前大气 $\mathrm{CO}_{2}$ 浓度已经达到 $380 \mu \mathrm{L} / \mathrm{L}$ (比工业革 命前的大气 $\mathrm{CO}_{2}$ 浓度 $280 \mu \mathrm{L} / \mathrm{L}$ 增加了 $100 \mu \mathrm{L} / \mathrm{L}$ ，见 图 1), 并以每年大约 $2 \mu \mathrm{L} / \mathrm{L}$ 的速度增长. 如果化石燃 料燃烧继续占全球能源消费的 $85 \%$ 或更高, 且全球 人口继续增加, 加上传统的、贫穷的民族工业化, 全 球大气 $\mathrm{CO}_{2}$ 浓度的增长速率将会成倍甚至三倍.

就这点而言, 我们必须清晰地认识到, 化石燃料 的相对缺乏将不会阻止大气 $\mathrm{CO}_{2}$ 浓度的增加. 尽管
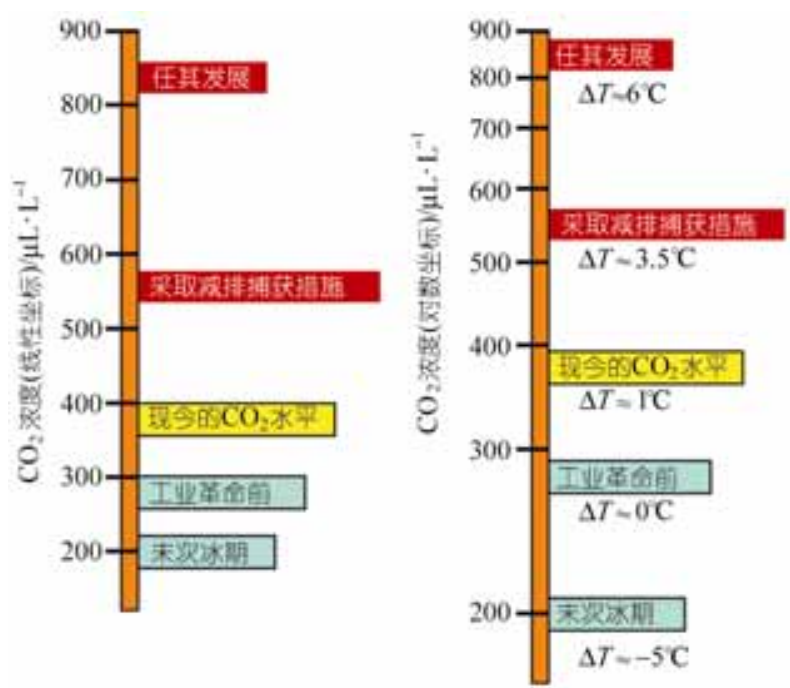

图 1 大气 $\mathrm{CO}_{2}$ 浓度过去(绿色)、现在(黄色)和将来(红色) 的变化情况

左侧表示浓度 $\mu \mathrm{L} / \mathrm{L}$, 右侧为与 $\mathrm{CO}_{2}$ 浓度相对应的温度变化. 右图为对数坐标

石油和天然气的储藏有限, 煤炭资源实际上还相当 可观. 此外, 煤炭转换为汽油经济可行, 交通燃料的 短缺将没有多少危机可言. 仅煤炭资源就能够满足 人类几个世纪的能源需求. 其他能源种类要形成与 煤炭资源的大范围竞争尚需时日. 例如, 目前太阳能 发电的成本大约是煤炭火力发电的 20 倍.

很多环境保护论者倡导大气中 $\mathrm{CO}_{2}$ 浓度不能超 过 $450 \mu \mathrm{L} / \mathrm{L}$ 的标准. 然而, 现实主义者声称, 即使付 诸巨大的努力, 人类也不能够阻止大气中 $\mathrm{CO}_{2}$ 浓度 上升至 $560 \mu \mathrm{L} / \mathrm{L}$ (即工业革命前的两倍). 悲观主义者 则担忧相关的重要措施不能被执行, 预测下个世纪 初期大气中 $\mathrm{CO}_{2}$ 浓度将达到 $840 \mu \mathrm{L} / \mathrm{L}$ (工业革命前的 3 倍).

要明白为什么把大气 $\mathrm{CO}_{2}$ 浓度控制在一定水平 之下(比如 $560 \mu \mathrm{L} / \mathrm{L}$ ) 是一个艰巨的挑战, 我们需要分 析控制化石燃料排放的各种限制. 一般而言, 每燃烧 40 亿吨碳, 大气 $\mathrm{CO}_{2}$ 浓度升高约 $1 \mu \mathrm{L} / \mathrm{L}$. 因此, 560 $\mu \mathrm{L} / \mathrm{L}$ 浓度所允许燃烧的最大碳量为 $40 \times(560 \sim 380)$ 亿 吨或 7200 亿吨. 就全球理想模式而言, 世界各国碳 分配按各国人口比例进行, 在这种情况下, 世界传统 工业化国家所分配到的碳排放量仅为总量的 $20 \%$, 约 1440 亿吨(见图 2). 目前, 所有这些工业化国家每 年消耗的碳量约为 60 亿吨, 如果保持这种速度, 在 短短 24 年内就会达到分配的排放额 ! 


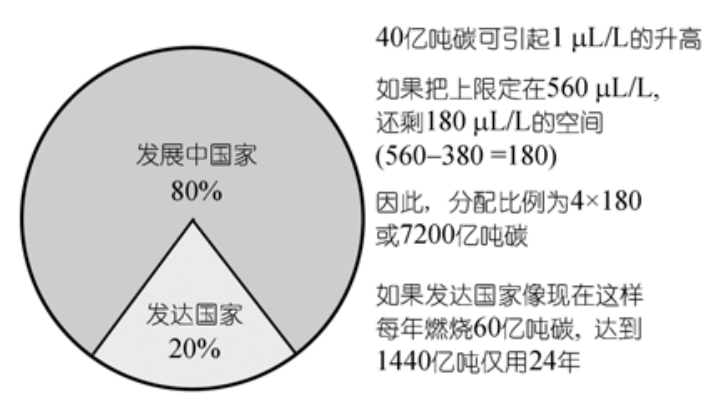

图 2 基于人口比例的碳分配

有关全球升温的争论并不关注未来 $\mathrm{CO}_{2}$ 浓度升 高的不确定性, 而是关注浓度升高后对气候产生的 未知影响. 利用世界超级计算机的强大计算功能, 先 进模型已经模拟出很多未知的结果. 尽管这些模型 如实地囊括了气候系统的每一个方面, 但很多情况 下气候系统的物理细节是模糊的, 因此模型设计者 们不得不借助经验参数来弥补这种空缺. 这些参数 中, 很多参数和水文循环密切相关(如水蒸气、云、降 水、海冰等).

\section{2 水汽反馈效应}

大部分对模型进行的气候预测结果的批评与所 谓的“水汽反馈效应”有关. 就自身而言, 大气 $\mathrm{CO}_{2}$ 浓 度的倍增将使全球气温升高 $1.2^{\circ} \mathrm{C}$. 然而, 由于全球 变暖, 液态水的水汽压将会相应升高, 从而使空气能 够容纳更多的水汽. 由于 $\mathrm{CO}_{2}$ 分子、 $\mathrm{H}_{2} \mathrm{O}$ 分子吸收地 球向外辐射(红外辐射), 在所有数值模型中, 随大气 $\mathrm{CO}_{2}$ 浓度的倍增而导致大气中水汽的增加将使全球 升温效应扩大 $2 \sim 3$ 倍 ${ }^{[2]}$. 因此, $1.2^{\circ} \mathrm{C}$ 就变成 $2.4 \sim 3.6^{\circ} \mathrm{C}$. 此外, 模型输出的内陆增温幅度将高于平 均增温, 且在北极海洋及其周边陆地是平均增温的 2 倍.

Richard Lindzen, 一位杰出的大气学家, 极力否 认这种水汽反馈效应将会在真实世界中出现 ${ }^{[3]}$. 他认 为热带大气中水汽含量将升高, 但在热带沙漠地区, 空气将变得更加干燥. 由于全球沙漠地区较低的水 汽含量, 并且缺乏云层遮盖, 沙漠地区大气将充当重 要的 散热器” 作用, 使地球排出太阳产生的热量. 因 此, Lindzen设想, 大气 $\mathrm{CO}_{2}$ 的累积不会放大全球升温 效应, 热带极端干旱区水汽的下降将抵消湿润地区 水汽含量的升高. 所得结果将是一个负反馈效应, 从 而削弱由于 $\mathrm{CO}_{2}$ 浓度升高所产生的全球气温升高. 正 如Lindzen的反对者所指出的一样, Lindzen因其观点
的与众不同而闻名, 例如他曾极力否认吸烟能够导 致肺癌.

\section{3 云和气溶胶}

计算机模拟中另外一个备受争议的方面是云. 云在地球辐射平衡中有重要作用, 因为云中小水滴 能够截获太阳入射光和地球向外的辐射. 云层范围、 位置或高度的微小变化将对气候模型产生显著的影 响 ${ }^{[4]}$. 另一个事实导致更大的复杂性, 即云层反射率 依赖于云中水滴平均尺寸的大小, 较小尺寸的水滴 一般比较大尺寸水滴的反射率大. 云层中小水滴的 平均尺寸依赖于所谓的云结核的有效性, 云结核越 多, 水滴数目也越多 ${ }^{[5]}$, 小水滴越多, 平均尺寸就越 小. 气溶胶可使有效性云结核的数目大大增加, 可能 使得云层变得更亮(见图 3).

在和水汽相关的要素中, 气溶胶是第二个重要 因素. 如温室气体一样, 气溶胶能够吸收地球向外的 红外辐射并再次辐射出来 ${ }^{[6]}$, 并且, 气溶胶也能像雨 滴一样散射太阳入射光. 和温室气体不同的是, 气溶 胶产生的影响用模型模拟较为困难. 一个原因是由 于气溶胶并不是均一地围绕在地球表面, 而是呈斑 块状并发生平移. 更为重要的是, 尽管颜色发亮的硫 酸盐气溶胶(通过氧化煤炭燃烧释放的 $\mathrm{SO}_{2}$ 而形成)能 够大大吸收太阳入射光, 但颜色发暗的气溶胶(由煤 炭和植物体燃烧形成)主要吸收地球向外辐射. 更为 复杂的是, 这些气溶胶之间相互碰撞, 合并形成更为 复杂的复合物. 此外, 所有这些气溶胶能够作为云结

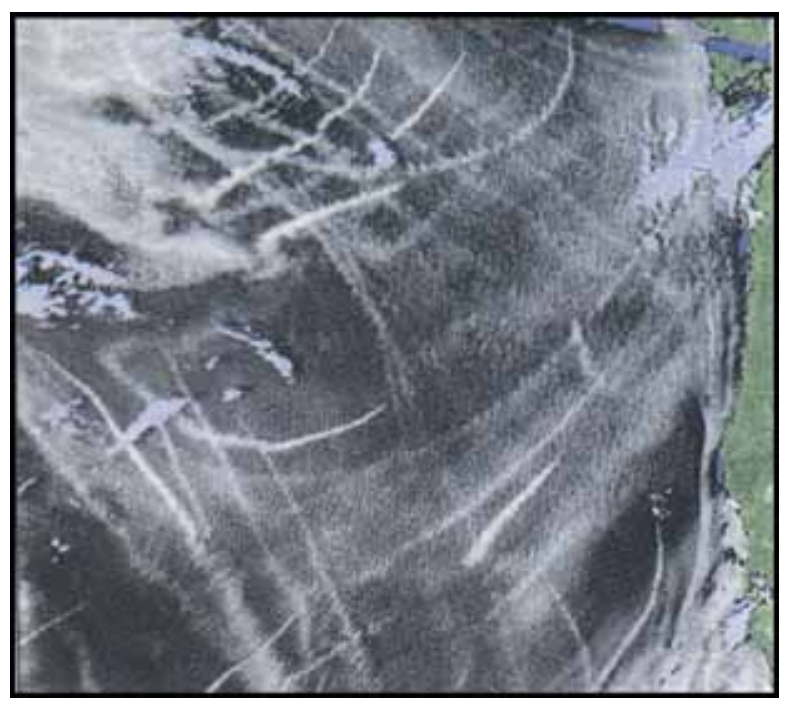

图 3 美国西海岸低云覆盖区航空照片 /亮线条由云结核产生(云结核通过下方区域船只排放的烟传递到云层) 
核. 尽管人为产生的气溶胶对气候过程产生影响是 无可置疑的, 但我们还并不清楚这些作用的结果到 底是阻碍还是促进了全球增温.

从长远来看, 空气中的气溶胶能够阻碍大气 $\mathrm{CO}_{2}$ 浓度持续升高的影响吗? 就目前的影响而言, 尽管 存在不确定性，但应该确信的是，随着时间的发展， 气溶胶的重要性将降低. 一个原因是由于气溶胶在 空气中驻留的时间只有几天到数周, 之后就被雨滴、 树叶等截获或带走. 相反, 人类活动释放到大气中的 $\mathrm{CO}_{2}$ 存留时间长达一个世纪, 第二个原因是气溶胶能 够构成健康威胁, 城市环境中气溶胶的形成将被大 幅度地减少. 因此, 到最后 $\mathrm{CO}_{2}$ 的作用当占上份.

\section{4 计算机模拟}

如果接受计算机模拟的结果, 大气 $\mathrm{CO}_{2}$ 浓度倍增 将导致气候变化, 最终影响人类的活动, 并造成对野 生动植物的有利生存环境发生较大的偏移. 但是, 每 一位气候学家都认为, 这些预测存在很大的不确定 性. 为了评价这些参数可能产生的偏移, 英国一个科 学家小组进行了周密的科学实验, 以评价模型对选 择参数的敏感性 ${ }^{[7]}$. 最后, 这个小组构造了几千个运 行模式, 对大气 $\mathrm{CO}_{2}$ 倍增情况下各种可能参数组合进 行检测. 这项实验得到了一系列的结果, 和正常参数 组合所得的结论相比, 这些结果更趋向于全球温暖 化. 而且这项实验中, 没有一个模式产生的增温幅度 低于 $1.5^{\circ} \mathrm{C}$. 这些实验表明, 模型的模拟结果很可能
低估了大气 $\mathrm{CO}_{2}$ 浓度升高的影响, 而不是高估.

表面上, 检测这些模拟结果可靠性的简便方法就是 确定他们是否正确地输出了温度计记录的全球温度 数据. 因为自工业革命以来, 大气 $\mathrm{CO}_{2}$ 浓度已经升高 了 $100 \mu \mathrm{L} / \mathrm{L}$ (从 $280 \mu \mathrm{L} / \mathrm{L}$ 到 $380 \mu \mathrm{L} / \mathrm{L}$ ). 此外, 甲烷和 其他红外吸收气体浓度的增长形成了额外的温室效 应, 这种效应几乎和 $\mathrm{CO}_{2}$ 浓度增长的作用相当. 因此, 这些温室效应的综合相当于 $\mathrm{CO}_{2}$ 浓度升高到 450 $\mu \mathrm{L} / \mathrm{L}$. 模型预测到 $\mathrm{CO}_{2}$ 浓度如此的增长应该使全球 温度升高约 $1.5^{\circ} \mathrm{C}$, 但事实如此吗?

\section{5 仪器记录}

图 4 表示地球表面年平均温度的变化趋势 ${ }^{[8]}$. 从 图 4 可以看出, 过去 140 年全球温度大约只升高了 $1.0^{\circ} \mathrm{C}$, 其中大约一半的增温发生在 1940 年以前(即 增温发生在温室气体显著增长以前). 1940 年至 1975 年间, 全球温度大致保持平稳, 仅在 1975 年后, 温度 才出现显著升高, 这可能归结于人为污染物的排放.

这种对比确定无疑地告诉我们, 人类活动导致 的全球温度变化高于地球自身所发生的变化. 很明 显, 19 世纪末 20 世纪初全球升温可能归结于自然因 素. 如果真是如此且模型模拟相同, 20 世纪中期全球 温度的平稳表明出现了自然冷确现象. 意外的是, 这 种自然冷却被人为导致的升温所抵消, 到 1975 年后, 人为影响才真正占据优势.

这种变化对反对者来说, 无疑是一个好消息. 他

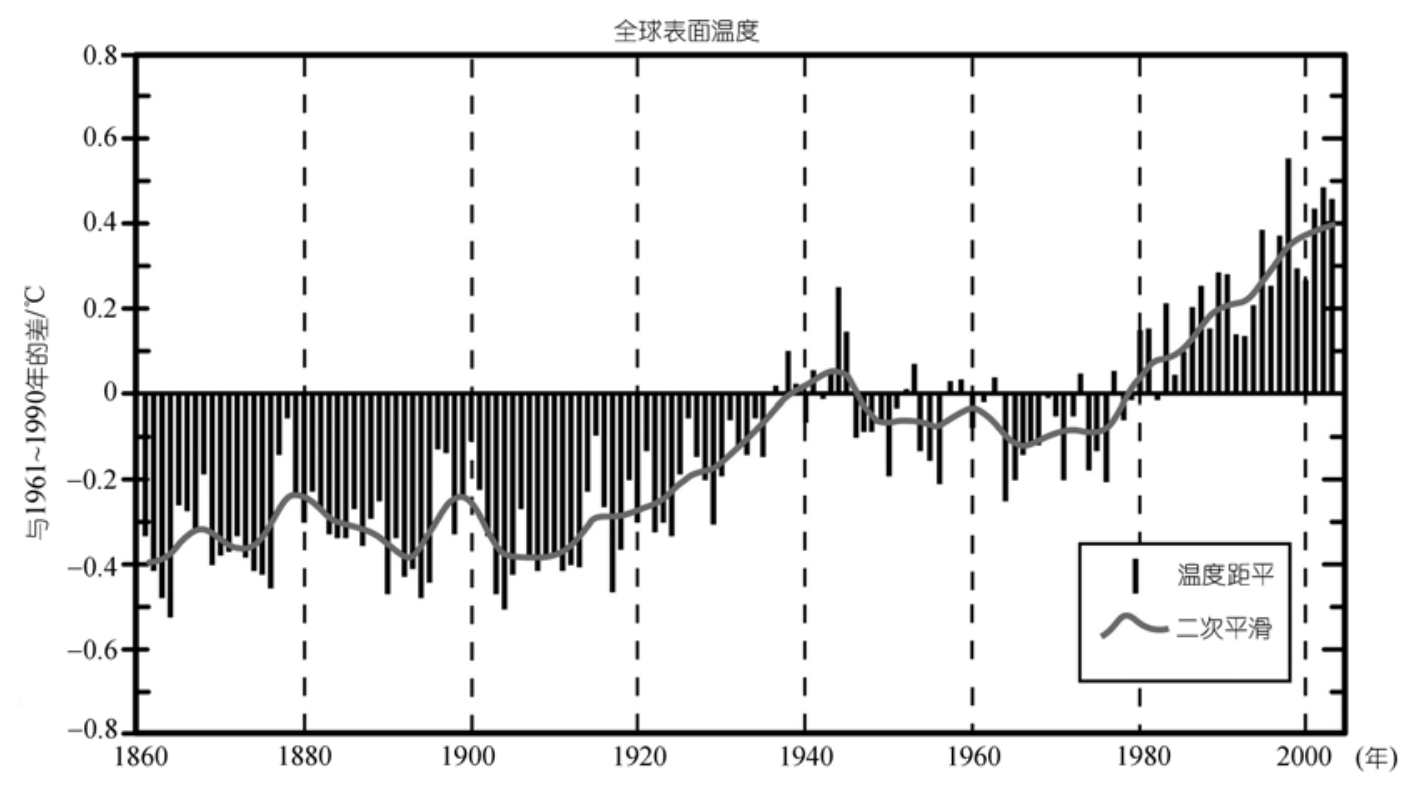

htt图: 4en全球各地区温度计记录的年平均温度 ${ }^{[8]} 343$ 
们能够说，也许所有警告都是正常的. Lindzen 也是 正确的. 沙漠空气中水汽的不足已经很大程度上抵 消了 $\mathrm{CO}_{2}$ 和其他温室气体的影响.

行动措施的支持者们将会受到反对浪潮的对抗！ 显然, 人为导致的升温已经使地球温度超出自然“噪 音”的范围. 正如我们无法预测在没有出现工业革命 的情况下地球温度将如何变化一样, 这种实验也是 无效的. 此外, 因为大气增温没有表层海洋快, 所以 在响应上存在明显的滞后. 浅色的气溶胶及由此组 成的云层对太阳光的反射可能抵消相当一部分由温 室气体所导致的升温.

因此, 在一定程度上, 这是一个平衡的局面. 那 些拒绝接受计算机模拟结果的人们, 能够使用温度 记录作为借口而不采取措施, 等到温室气体产生显 著的升温效应后再采取昂贵的措施. 而那些把模型 预测结果作为未来导向的人们则会认为, 如果不制 定削减大气 $\mathrm{CO}_{2}$ 浓度的计划并尽快付诸行动, 我们 将没有机会阻止 $\mathrm{CO}_{2}$ 浓度攀升至 $560 \mu \mathrm{L} / \mathrm{L}$. 为了阻止 $\mathrm{CO}_{2}$ 浓度的增长, 我们不得不对整个能源基础设施进 行全面的改造.

\section{6 古气候记录}

幸运的是，在我们的估测中存在另外一个信息 来源, 这些信息倾向于支持行动者的一方. 这就是储 藏于冰盖、沉积物和石笋中的古气候记录. 为了把储 存于这些载体中的信息联系起来, 古气候研究者开 发了精确的方法进行全面的测年, 只有采用这种方 法, 才能够把世界不同地区的古气候记录联系起来. 这项任务类似于历史学家, 通过日历把发生在不同 国家的事件联系在一起. 除这种时钟方法外, 这些物 体中必须包含记录温度、降水、冰川范围、大气 $\mathrm{CO}_{2}$ 浓度的代用指标. 过去 50 年来, 得到了时钟和代用 指标的惊人序列 ${ }^{[9]}$, 把两种方法所得结果综合在一起, 得到了地球过去 50 万年气候演变图. 对我个人而言, 气候重建的作用是显而易见的, 地球气候系统并不 是对微小变化没有反应, 而是反应强烈. 过去较大的 气候变化都是由环境的微小变动所导致. 与此相比, 目前大气 $\mathrm{CO}_{2}$ 浓度的成倍上升不仅仅是微小的环境 变动, 而是一次大的环境因素变化.

对过去 50 万年气候记录重建的方式不必赘述. 这里仅简要阐述地球所经历的三种气候变化. 三种 变化中的每一种都可视为是对已知的小的触发因素 的强烈反应.
例 1: 过去 50 万年中, 共出现了 4 次大的冰期. 每次都包括北美和欧洲冰盖的发展. 在北美, 冰川覆 盖了现在的整个加拿大, 向南深入到美国 Ohio 河及 Staten 岛. 由于冰盖的发育, 海平面和现在相比大约 下降了 $120 \mathrm{~m}$. 南极冰芯的气泡记录告诉我们, 每次 冰期大气 $\mathrm{CO}_{2}$ 浓度可下降至 $190 \mu \mathrm{L} / \mathrm{L}$. 运用模型对未 来进行模拟, $\mathrm{CO}_{2}$ 浓度的下降能够解释冰期时好几度 的降温. 在这四次旋回中, 每个旋回的状况都基本相 同，即持续 10 万年的降温和冰盖扩张，在升温中达 到顶点, 并突然地结束冰期而使地球返回到间冰期 阶段. 过去 1 万年是人类文明发展的阶段, 地球恰恰 处于这样一个间冰期(见图 5).

叠加于上述气候恶化上的是突出的 2 万年周期 (见图 5). 这些周期的存在提示我们, 导致冰期的因 素在时间上与太阳辐射季节分配变化完美地同步. 这些周期性变化, 包括冬夏太阳辐射反差的增强和 减弱, 是地球轨道岁差变化的结果. 包括除 2 万年周 期外, 地球轨道对气候影响的证据来自于每 10 万年 冰期的突然终止, 每次均发生于冬夏差异最大的时 间. 地球轨道变化和气候变化的对应让人信服一个 现在被广为接受的观点, 即冰期周期与季节差异变 化有关. 本文想强调的一点是, 当这些季节变化引入 到常用来预测温室气体气候效应的模型时, 模型几 乎没有响应. 这告诉我们, 在客观世界中肯定发生着 一些相互作用(模型中没有), 显著地放大了一些与太 阳热量季节分配相关联的因素的影响.

例 2: 古气候学家对这样的情况很难解释, 即南 极冰盖长冰芯记录的气温和大气 $\mathrm{CO}_{2}$ 浓度的变化(见

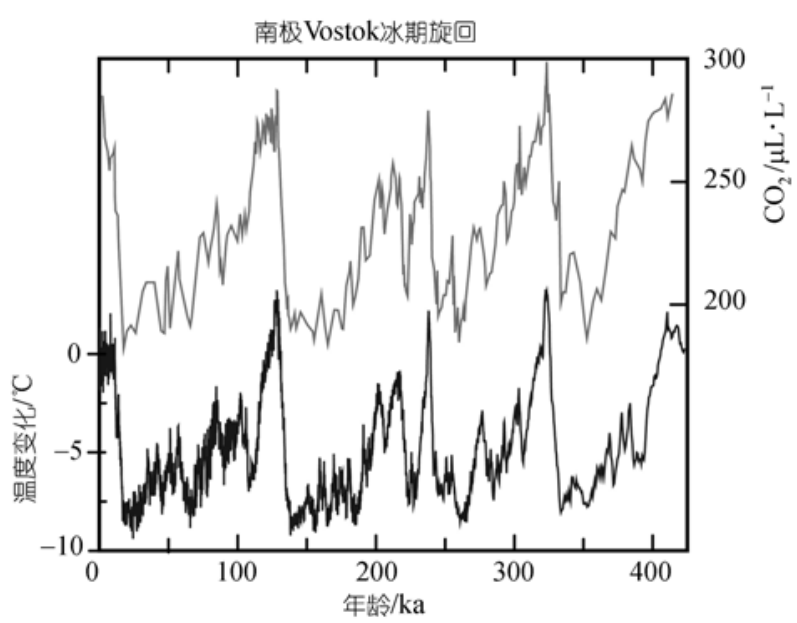

图 50 . 南极Vostok冰芯大气 $\mathrm{CO}_{2}$ 浓度记录和大气温度记录 
图 5)与地球轨道周期的期望值非常吻合, 但格陵兰 冰芯的记录却完全不同. 如图 6 所示, 后者记录的变 化以千年尺度的跳动为主. 在最后一次冰期旋回中, 大约发生了 20 次这样的反复变化 ${ }^{[11]}$. 这种变化不仅 出现在温度记录中, 而且出现在冰芯的沙尘记录中 [12]，冰芯气泡的甲烷记录中也有所体现 ${ }^{[13]}$. 因为格 陵兰冰芯有显著的年层, 所以能够显示出每次这样 的变化都以较快速度发生, 即在短短几十年内完成. 与这些突变相关联的年平均温度的变化都在 $10^{\circ} \mathrm{C}$ 以 上 ${ }^{[14]}$.

这一发现促使科学家在地球其他地方寻找相同 的记录. 在广泛的北方温带和热带地区, 发现了气候 快速变化的现象, 但在南半球, 即使出现这种现象,

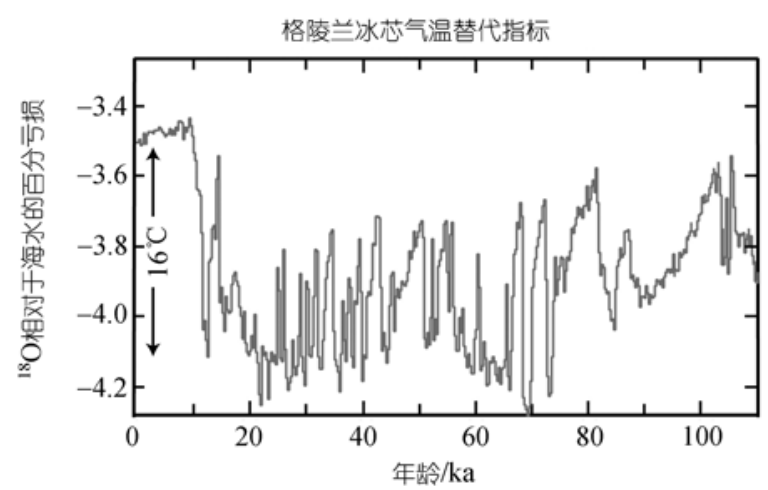

图 6 格陵兰Summit冰芯记录的大气温度变化 ${ }^{[11]}$
变化也较弱, 并且在时间上有所偏移. 尽管从最初就 推断这种偏移是由大西洋的洋流重组引起(见图 7), 但约 20 年以后, 才发现这种突变及其广泛分布的地 理作用, 最关键的一点是由于北大西洋海冰大规模 变化的巨大放大作用 ${ }^{[16]}$, 这些变化的出现响应于大 西洋环流输送带的开始和结束. 当输送带开始时, 北 部环流的上部分支流经热带地区, 海水被加热, 最后 被加热的海水流入挪威海. 但当输送带停止时, 这种 热带地区的热量传递不再存在, 导致冬季海冰能够 覆盖北大西洋, 向南深入到英伦列岛. 这些海冰不仅 阻碍海洋热量向大气的传递, 还可能反射大部分入 射太阳光. 所以, 欧洲冬季几乎能够和西伯利亚 相当.

但是, 寻找和热带地区的联系是一个最大的挑 战. 来自中国洞穴和阿拉伯海沉积物的气候记录明 确显示出, 季风在海冰覆盖北大西洋时被大大减弱. 此外, 距离南美洲加勒比海岸不远的 Cariaco盆地沉 积物以及巴西东南部洞穴石笋的气候记录都表明, 当海冰覆盖北大西洋时期, 热带降雨带向南偏移了. 北大西洋海冰引入到模型中, 既能够说明季风减弱 的原因, 也能够说明热带降雨带向南偏移的影响因 素 ${ }^{[16]}$. 后者反映了由于北部剧烈降温所导致的热带 赤道向南平移. 前者是由北部温带地区长时间的积 雪所引起, 这种长时间的积雪延迟了亚洲的夏季变

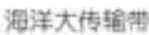

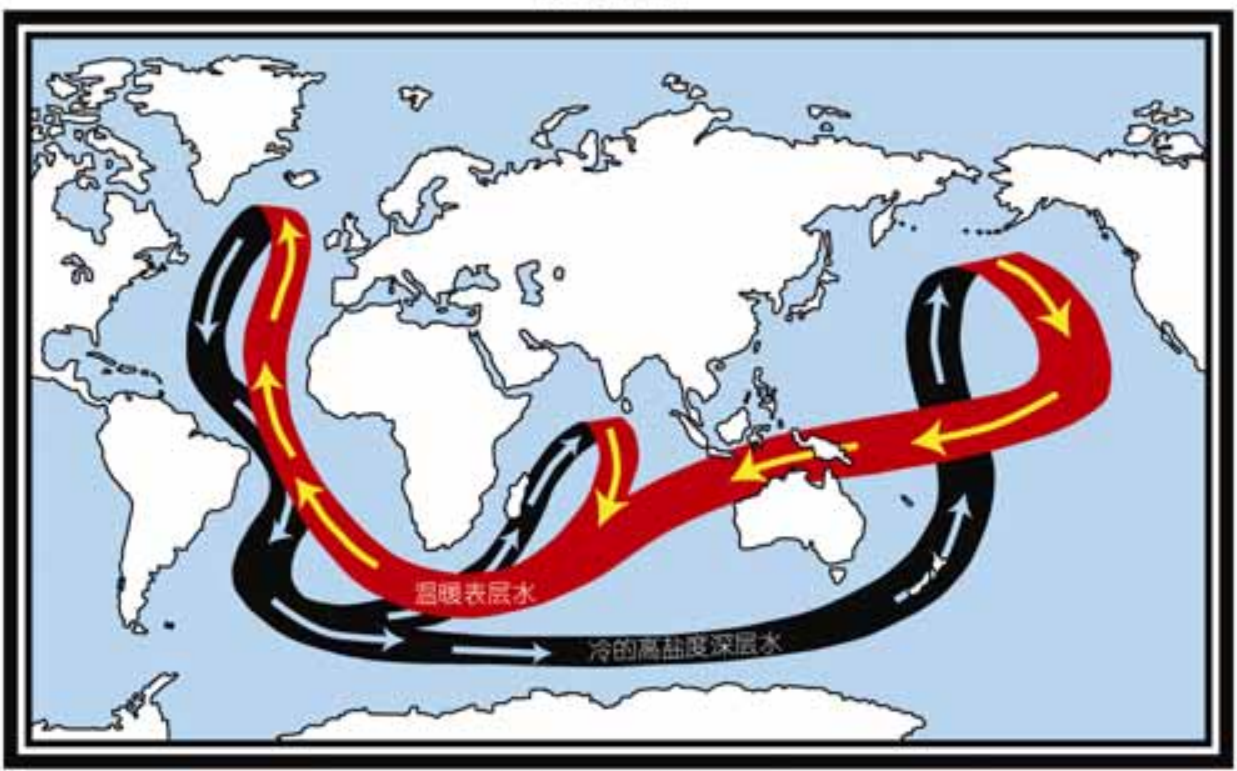

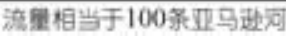

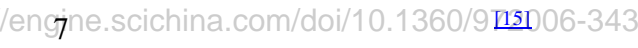


暖, 因此减弱了季风 ${ }^{[17]}$.

第二个实例不仅揭示了自然界潜在的放大效应, 还表明海洋和大气之间的相互作用引起了所谓的气 候离散量子状态(discrete quantum states of climate). 地球系统能够在这些离散状态之间发生突变, 这种 突变不仅对温度和降水产生深远影响, 还对大气中 $\mathrm{CO}_{2}$ 和 $\mathrm{CH}_{4}$ 含量产生重要影响.

例 3: 与末次冰期相比, 目前所处的间冰期气候 表现很好, 仅 8200 年前, 大西洋环流输送带停止了 [18]，但没有持续几个世纪，而是在几十年后就又恢复 了功能. 令人感兴趣的是, 计算机模拟产生的结果是 一系列温度的短周期变化 ${ }^{[19]}$, 这些变化很好地记录 于高纬度地区. 每一个完整的周期变化, 温度变幅大 约为 $1{ }^{\circ} \mathrm{C}$, 持续时间大约为 1500 年. 中世纪变暖 (800 1350 AD)和小冰期(1350 1850 AD)是这一序列 中距今最近的事件. 这些周期变化发生的时间和大 气中两个放射性同位素— $-\mathrm{C}^{14}$ 和 $\mathrm{Be}^{10}$ 产生速率的时 间周期紧密相应 ${ }^{[19]}$. 放射性同位素产生速率的变化 反映了银河系高能量宇宙射线进入大气的变化. 这 种变化是源于太阳黑斑(如所谓的太阳风)中离子所 产生磁场强度变化的结果. 太阳黑斑越多, 外逸离子 就越多, 离子产生的磁场强度就越强. 卫星观测资料 表明, 在过去两次 11 年的太阳黑子活动周期中, 太 阳发光度已经发生了微小的变化(见图 8). 过去两次 太阳黑子活动高峰期和其间太阳黑子活动低谷期相 比, 太阳辐射输出量前者比后者高 $1 / 1300^{[20]}$. 即使人 类肉眼观测太阳黑子仅追溯到 1604 年(最先被伽利略

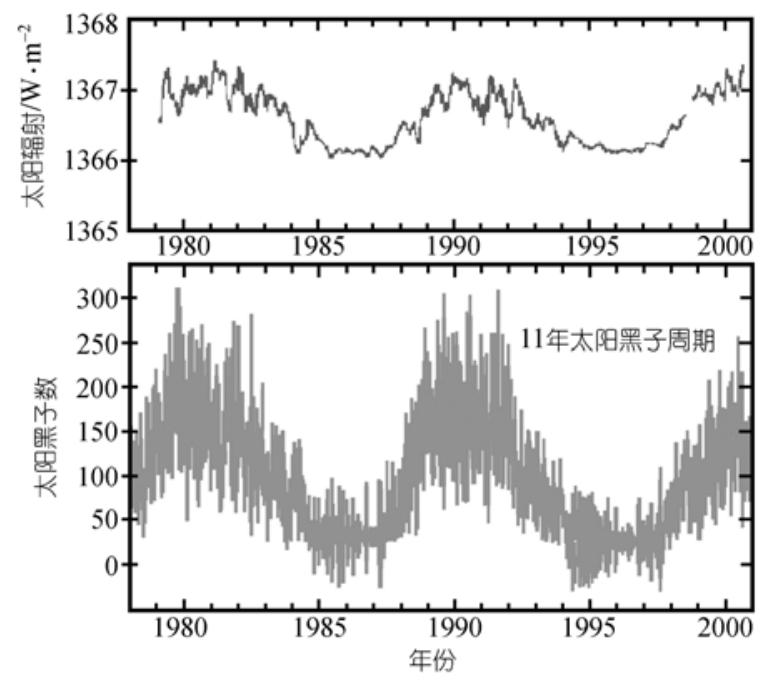

图 8 卫星测量的最后两个 11 年太阳黑子周期期间 的太阳辐射 20
发现), 但通过同位素得到的替代指标却能够扩展到 整个全新世(即目前的间冰期). 难以理解的是, 至今 没有发现好的途径来解释太阳发光度在 $1 / 1000$ 数量 级上的变化能够导致 $1^{\circ} \mathrm{C}$ 的温度波动. 根据模型模拟, 这种数量级的发光度的变化能够产生小于 $0.1^{\circ} \mathrm{C}$ 的温 度波动 ${ }^{[21]}$. 因此, 我们再一次证明了地球气候系统具 有潜在的放大作用, 能够对微小的波动产生强烈的 响应.

地球轨道周期性变化产生的季节变化或太阳辐 射的微小变化都能对地球气候产生影响. 这些影响 不能利用模型进行准确模拟, 表明这些模型也可能 低估 $\mathrm{CO}_{2}$ 和其他温室气体产生的长远影响. 此外, 海 洋环流变化导致的大幅度突发性变化也警示我们, 进入未来温室气候的过程可能经历大的波动! 古气 候记录使我确信, 气候变化类似一头发怒的野兽, 当 受到攻击时会做出激烈的响应. 因此, 我深信我们应 采取一切行动来减小温室效应的激发作用!

\section{7 末来环流输送带会停止吗?}

人们已经广泛关注这些观点, 即全球升温将导 致大西洋环流输送带的停止, 欧洲也将受到另外一 个冰期的袭击. 我个人认为, 这些观点过于夸大. 模 型模拟确实表明, 温室效应诱发的过量降水以及河 水涌入北极和北大西洋能够稀释表层海水含盐量, 低到一定程度后导致环流输送带停止. 但是, 这并不 是一个突然的开关过程, 而是类似于冰期所导致的 气候变化, 模型模拟表明, 这种逐渐的、缓慢的变化 需要大约一个世纪 ${ }^{[22]}$. 此外, 为了产生足量的额外淡 水输入, 模拟模型得出地球温度须升高 $4 \sim 6^{\circ} \mathrm{C}$, 大气 中 $\mathrm{CO}_{2}$ 浓度必须达到工业革命前水平的 3 倍! 升温到 这样的程度, 环流输送带的停止就没有可能促使北 大西洋海冰的形成. 没有海冰引发的放大效应, 输送 带停止将失去大部分激发作用. 这样就不是另外一 个欧洲冰期的开始, 而可能是部分抵消了温室气体 产生的增暖效应.

假设未来环流输送带逐渐停止, 类似于冰期中 的和 8200 年前的事件怎样突然发生? 回答是, 过去 数个这样的事件确有记录, 是由淡水大量涌入北大 西洋引起的. 淡水涌入的一种模式是集中在Hudson 海湾的冰盖坞塌, 使得冰山侵入北大西洋, 大量冰山 融化后增加了大量的淡水 ${ }^{[23]}$; 另一种模式是由于加 拿大冰抽退缩, 积累于湖泊中的大量淡水形成巨大 
的洪水 ${ }^{[24]}$. 如今, 只有格陵兰冰盖拥有能够引发海洋 环流突然终止的谈水量. 然而, 目前没有一个关于格 陵兰对全球升温的相应模型显示冰盖能够发生瞬时 的大规模坍塌进入海洋, 而是显示高纬度地区降水 量将缓慢上升, 格陵兰冰盖的融化速率也将缓慢 升高.

\section{8 有关水危机}

如果上述估测是正确的, 我们能够设想温室效 应一路上不会发生任何大的冲击吗? 这个可能也许 有, 但就过去 50 万年来说, 地球气候没有经历过比 今天更为显著的温暖, 我们也许正在进入一个未知 的境界. 我猜测这些将包括降水的重新分布(即干旱 和洪水). 气候模型表明, 随着全球变暖, 热带地区降 水将更多, 而荒漠地区降水将更少. 尽管我们缺乏全 球升温的相似型, 但有研究很好的降温相似型. 在冰 期时，北美西部、中东、中国西北部以及澳大利亚荒 漠中封闭的盆地湖泊是现在的好几倍大. 而热带地 区这样的证据很少. 末次冰期时Victoria湖发生干涸 [25], 非洲大裂谷中其他湖泊比现在也小得多. 这 样, 似乎当全球变冷时, 热带地区的降水就更少, 相反沙 漠地区的降水就更多. 所以, 古气候记录增加了
模型预测的可信度.

但是, 有人可能会问, 湿度的这些变化是逐步的, 还是突然的? 尽管这个问题没有肯定的答案, 但目 前间冰期发生的区域干旱提供了一些重要的线索.

在这些干旱中, 最剧烈的两次发生于中世纪温 暖期的北美西部地区, 它们被死亡树木的中继线完 好地记录下来, 反映了该地区沙漠西部边缘过去的 湖泊、河流及沼泽水位. 年轮统计表明部分树木存活 了 150 年. 放射性碳测年表明这两次剧烈干旱发生在 1050 1200 AD和 1300 1375 $\mathrm{AD}^{[26]}$. 格外有趣的是, 树木中断线推测了 Yosemite 峡谷附近高海拔地区 Tenaya湖的水位线. 这些树木扎根深度至 $13 \mathrm{~m}$ 以下 基岩, 充当湖泊的出水口. 过去 130 年来, 这个湖泊 仅有一次没有溢出由于冰雪融化侵入的淡水. 在中 世纪暖期两个世纪内, 这个湖泊从没有被充满过. 相 反, 减少的入湖水量一定被全部蒸发了. 我们能够确 對的说, 如果湖泊水位上升到基岩水平并且外溢, 湖 水将会杀死树木.

许多其他地点的树木中继线也记录了类似的现 象(见图 9). 显然, 西部干旱区经历了长达两个世纪 的干旱, 这比过去 100 年内发生的三次持续时间为 8

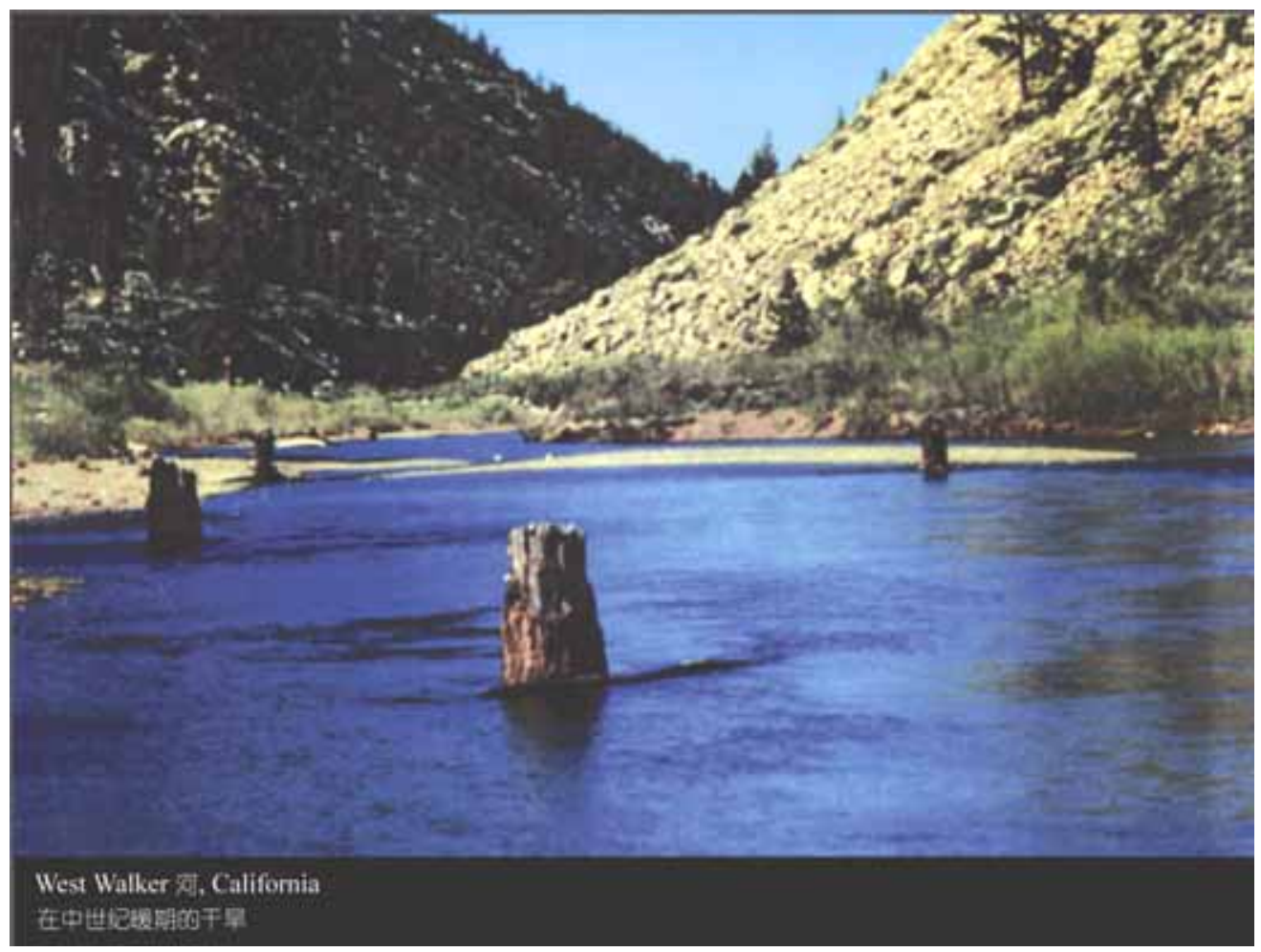

图 9 第一次中世纪干旱期生长于 West Walker 河床的树体残留(courtesy of Scott Stine, California State University, Hayward) 
年的任何一次干旱都要剧烈. 就此而言, 有一点是令 人吃惊的, 即中世纪温暖期的气候并不比现在显著 温暖.

但是,一个悬而未决的问题是, 发生的几次干旱 是否突然的开始和结束? 目前, 我们仅能说, 两次干 旱被位于加利福尼亚Sierra Nevada 山脉东部沙漠中 的封闭盆地Owens 湖和Mono 湖的高水位事件隔开 [27]. 目前, 正在进行相关研究, 以期确定树木年轮宽 度对这几次大干旱发生与结束的响应.

\section{9 总结}

我列举的案例很硬, 但并非无懈可击. 因此, 前 述的反对者可能以此认为可以延迟采取行动, 除非 全球升温明显的高于自然变率. 如果有一个设计良 好的路线图, 清楚的说明阻止大气 $\mathrm{CO}_{2}$ 浓度升高的 方法和时间表, 我可能会赞成. 但是, 我们是在和时 间赛跑, 不能坐以待毕. 我们至少需要 20 年来研究 制止 $\mathrm{CO}_{2}$ 浓度升高的方法, 也至少需要 40 年甚至更 多的时间来执行这些方法. 然而，现在不仅没有这些 方法, 甚至什么是关键问题都没有达成一致. 此外, 多种迹象表明, 即使现在马上采取行动, 我们也难以 阻止大气 $\mathrm{CO}_{2}$ 浓度上升到工业革命前 2 倍的水平.

\section{0 我们必须做些什么?}

30 多年来, 全球温度持续稳定增长, 但阻止 $\mathrm{CO}_{2}$ 浓度升高的成效甚微. 无可置疑, 京都议定书已开始 生效, 但这仅仅是控制大气中温室气体浓度增加漫 长过程中的第一步. 由于世界经济发展导致化石燃 料消耗的增长, 即使那些签约国实现减排许诺, 每年 大气中 $\mathrm{CO}_{2}$ 浓度的增长速度也将比以前快. 事实上, 风能已经发挥作用, 但即使出现最好的局面, 其产能 也不超过全球能源的 $10 \%$. 核能正在恢复, 但世界充 满恐怖主义, 几乎无人相信核能能够取代化石燃料 成为将来最主要的能源. 太阳能发电是很多人梦棩 以求的解决方法, 但也只能维持现状, 除非其价格能 下降一个数量级.

我认为, 无论我们怎样推进能源储存, 促进发展 可再生能源, 化石燃料燃烧也将持续增长. 大作宣传 的氢能源经济也注定不是万能药, 因为制氢设备所 需能量使用煤炭比使用电解水要便宜 10 倍. 太阳能 发电直到成本下降后才能形成竞争. 同样, 核能发电 也在等待最佳的经济成本.

可能有些人会说, 不要着急,
全的核能、太阳能等结合起来, 使我们脱离困境. 但 如果这些不成怎么办? 我们不应该拥有一个好的后 备计划吗? 值得庆幸的是, 这里有一个可行的后备 策略, 允许我们从化石燃料中发展能源而不增加大 气 $\mathrm{CO}_{2}$ 浓度. 换言之, 我们能够拥有自己的蛋糕并分 享它!

这个策略就是捕获 $\mathrm{CO}_{2}$ 并埋藏. 一个方法是从发 电厂排气装置中捕获 $\mathrm{CO}_{2}$, 但目前由于我们消耗能量 的 $2 / 3$ 产自于小个体(汽车、家庭、工厂、飞机等), 所 以捕获是不可能完全的. 除此之外, 不得不从大气中 捕获 $\mathrm{CO}_{2} \stackrel{[28]}{\text {. }}$.

$\mathrm{CO}_{2}$ 捕获策略能够以合理的成本实行吗? 答案 是肯定的. 就煤炭发电厂而言, 如果煤炭进行熏蒸产 生氢, 氢能够用来产生电力, 这可能是非常经济的. 原因在于, 从煤炭熏蒸产物中将氢与 $\mathrm{CO}$ 分离所需的 基础设备与传统燃煤发电厂从废气中清除 $\mathrm{CO}_{2}$ 所需 的设备相比, 前者要便宜的多.

尽管从大气中俘获 $\mathrm{CO}_{2}$ 的想法初看起来似乎是 一个不可能完成的挑战, 然而这个想法是可行的. 让 我们来看看其可行性的原因, 把一股假定的风流所 具备的动力学能量和其携带的 $\mathrm{CO}_{2}$ 量相比, 听起来 像一个苹果和一个柑桔的比较, 但事实并非如此. 气 流携带的 $\mathrm{CO}_{2}$ 量能够被看成产生这些 $\mathrm{CO}_{2}$ 燃烧的化 石燃料的量.

从这个角度去考虑, 就是能量与能量之间的比 较. 这种对比表明 $\mathrm{CO}_{2}$ 超过风动力的 100 倍! 此外, $\mathrm{CO}_{2}$ 能够使用基本的方法吸收, 并以纯的性状释放出 来, 允许吸收剂循环使用.

因此, 如果风的涡轮运动能够与燃煤电力发电 厂对比, 从大气中重新俘获 $\mathrm{CO}_{2}$ 可能是经济可行的 (见图 10). 实际上, 亚利桑那州 Tucson 市一个小公司 已经花费两年时间来生产此俘获设备的原型. 他们 希望能够在 2007 年进行实地测试, 并估计该设备的 成本将是产生这些 $\mathrm{CO}_{2}$ 能量的成本的 $15 \%$. 换言之, 该设备成本与消费同步, 一加仑汽油或一千瓦特电 的价格大概增长 1.15 倍.

当然, $\mathrm{CO}_{2}$ 一旦被捕获, 不管其来自能源工厂还 是直接来源于空气, 被捕获的 $\mathrm{CO}_{2}$ 必须储藏. 很多方 法被建议来储藏 $\mathrm{CO}_{2}$, 每种方法都有各自的成本、容 量和环境效果. 每种方法中, 第一步都是把气态 $\mathrm{CO}_{2}$ 转换为液态, 即在常温下把气态 $\mathrm{CO}_{2}$ 压缩 14 倍. 在 液态下,, 通过和传输石油同样的管道线, $\mathrm{CO}_{2}$ 能够被 


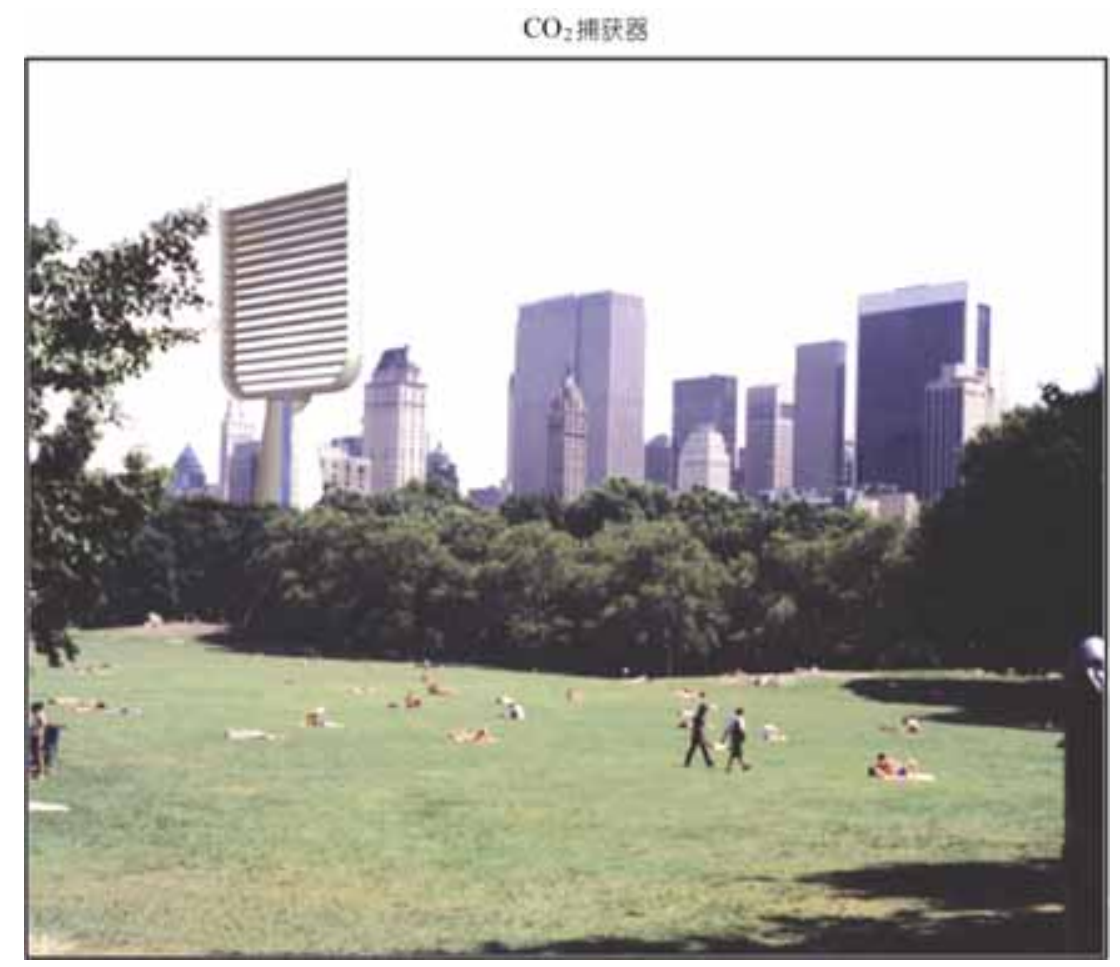

图 10 一种捕获大气 $\mathrm{CO}_{2}$ 设备的构思

转运到埋藏地点. 在这些处置方法中, $\mathrm{CO}_{2}$ 通常被埋 藏到咸的蓄水层中(位于沉积地层 1 2 km深处)、南极 冰盖底下的湖泊中、深海以及玄武岩连续性运动分离 的多孔层. 另外一个花费更高的方法就是挖掘后埋 藏在富含镁的可溶性硅酸岩中, 其中镁能够与 $\mathrm{CO}_{2}$ 反 应形成高度稳定的镁碳酸岩矿物一一方镁石 ${ }^{[28]}$.

少数工程已经启动. 一家挪威公司 (Statoil)正分 离来自于北海(North Sea)地下天然气中的 $\mathrm{CO}_{2}$. 这些 $\mathrm{CO}_{2}$ 被液化后, 压进地下天然储气层上部的储水层中 [28]. 英国石油公司(British Petroleum)正在计划使用 输送北海石油的管道输送煤炭燃烧产生的液态 $\mathrm{CO}_{2}$.

当然也要想到, 绿色和平组织极力反对把液态 $\mathrm{CO}_{2}$ 储藏至海底. 如果埋藏在南极冰盖底下的意见被 明确提议, 将可能出现更强烈的反对声。一般情况下 液态 $\mathrm{CO}_{2}$ 储藏至玄武岩层或碱性蓄水层(或形成方镁 石)不会使用地球的公共场所，同时这些埋藏地点服 从于每一个国家的权限.

毫无疑问, $\mathrm{CO}_{2}$ 捕获与储藏是阻止大气中 $\mathrm{CO}_{2}$ 浓 度升高计划中的最重要措施之一, 但在这项策略能 够大范围的执行之前, 我们需要付诸巨大的努力. 装 备有 $\mathrm{CO}_{2}$ 捕获设备的煤炭气化工厂必须建立起来并 进行检测, 大气 $\mathrm{CO}_{2}$ 捕获设备也需要建立等. 我们必
须实行储藏评价措施, 以确保 $\mathrm{CO}_{2}$ 泄漏达到最小化, 并确保不可能存在剧烈的泄漏现象.

此外, 我们必须出台一个方法, 通过该方法, 能 源使用者的付费能够顺利地转移至捕获和埋藏 $\mathrm{CO}_{2}$ 的公司. 最后, 世界各国间化石燃料燃烧释放 $\mathrm{CO}_{2}$ 的 分配必须达成一致. 一旦达成一致并开始执行, 将产 生一个方法来监控和督促各个缔约国的执行情况. 无疑, 这三个任务中最后的一个是迄今为止最为困 难的. $\mathrm{CO}_{2}$ 过去的排放量将怎样和未来排放量进行协 调排列呢? 经济发达国家应该负担多大比例的 $\mathrm{CO}_{2}$ 埋藏成本呢? 那些阻碍签约的国家怎样才能够成为 缔约的国家呢?

\section{1 结论}

我个人认为, 本文标题所提出问题的答案是, 阻 止全球变暖的措施刻不容缓. 地球气候系统已经显 示出对微小扰动的激烈反应能力. 我们泰然自若地 对地球产生了相当大的扰动, 必须慎重考虑采用合 理的手段阻止大气中 $\mathrm{CO}_{2}$ 浓度超过工业革命前的 2 倍. 尽管我们采用一切可能的办法来保存能源并采 用可更新资源, 但这些措施是不够的. 由于煤炭资源 的廉价性以及发展中国家对能源需求的不断增长性 
等现象的客观存在，尽管我们尽最大的努力, $\mathrm{CO}_{2}$ 排 放也将会持续增长. 如果成功地限制这种增长, 我们 需要利用 $\mathrm{CO}_{2}$ 捕获和埋藏技术来补充能源储藏和 更新.

致谢 本文得到 Jeff Severinghaus 和 Huan-Hui Li 的详细 评阅, 并得到哥伦比亚地球学院的资助. 中文稿由董云社 翻译, 郭正堂校对. 在此一并致谢.

\section{参考文献}

1 Quay P, Sonnerup R, Westby $\mathrm{T}$, et al. Changes in the ${ }^{13} \mathrm{C} /{ }^{12} \mathrm{C}$ of dissolved inorganic carbon in the ocean as a tracer of anthropogenic $\mathrm{CO}_{2}$ uptake. Glob Biogeochem Cycle, 2003, 17: doi: 10.1029/2001GB001817

2 Soden B J, Jackson D L, Ramaswamy V, et al. The radiative signature of upper tropospheric moistening. Science, 2005, 310: 841 -844[DOI]

3 Lindzen R S, Nigam S. On the role of sea-surface temperature gradients in forcing low-level winds and convergence in the tropics. J Atmos Sci, 1987, 44: 2440-2458

4 Slingo A. Sensitivity of the Earth's radiation budget to changes in low clouds. Nature, 1990, 343: 49—51[DOI]

5 Penner J E. The cloud conundrum. Nature, 2004, 432: 962963[DOI]

6 Hansen J, Sato M, Ruedy R, et al, Efficacy of climate forcings. J Geophys Res, 2005, 110: D18104, doi: 10.1029/2005JD005776

7 Stainforth D A, Aina1 T, Christensen C, et al. Uncertainty in predictions of the climate response to rising levels of greenhouse gases. Nature, 2005, 433: 403-406[DOI]

8 Hansen J, Ruedy R, Glascoe J, et al. GISS analysis of surface temperature change. J Geophys Res, Atmospheres, 1999, D24: 30997-31002[DOI]

9 Broecker W S. The Glacial World According to Wally. Third Revised Edition. New York: Eldigio Press, 2002

10 Petit J R, Jouzel D, Raynaud N I, et al. Climate and atmospheric history of the last 420,000 years from the Vostok ice core. Nature, 1999, 399: 429-437[DOI]

11 Stuiver M, Grootes P M. GISP2 Oxygen isotope ratios. Quat Res, 2000, 53: 277-284[DOI]

12 Mayewski P A, Meeker L D, Whitlow S I, et al. Changes in atmospheric circulation and ocean ice cover over the North Atlantic during the last 41,000 years. Science, 1994, 263: 1747-1751

13 Brook E J, Sowers T, Orchardo J. Rapid variations in atmospheric methane concentration during the past 110,000 years. Science, 1996, 273: 1087-1091

14 Severinghaus J P, Grachev A, Luz B, et al. A method for precise measurement of argon 40/36 and krypton/argon ratios in trapped air in polar ice with applications to past firn thickness and abrupt climate change in Greenland and at Siple Dome, Antarctica. Geochim Cosmochim Acta, 2003, 67: 325-343[DOI]

15 Broecker W S. The Great Ocean Conveyor. Oceanography, 1991, 4 : 79-89

16 Chiang J, Biasutti M, Battisti D S. Sensitivity of the Atlantic Intertropical Convergence Zone to Last Glacial Maximum boundary conditions. Paleoceanography, 2003, 18: 1094[DOI]

17 Barnett T, Dümenil L, Schlese U, et al. The effect of Eurasian snow cover on global climate. Science, 1988, 239: 504-507

18 Alley R B, Mayewski P A, Sowers T, et al. Holocene climatic instability: A prominent, widespread event 8200 yr ago. Geology, 1997, 25: 483-486[DOI]

19 Bond G, Kromer B, Beer J, et al. Persistent solar influence on North Atlantic Climate during the Holocene. Science, 2001, 294: 2130-2136[DOI]

20 Fröhlich C, Lean J. The Sun's total irradiance: Cycles, trends and related climate change uncertainties since 1976 . Geophys Res Lett, 1998, 25: 4377-4380

21 Douglass D H, Clader B D. Climate sensitivity of the Earth to solar irradiance. Geophys Res Lett, 2002, 29(16): doi: 10.1029/ 2002GL015345

22 Stocker T F, Knutti R, Plattner G-K. The future of the thermohaline circulation — A perspective. In: The Oceans and Rapid Climate Change: Past, Present, and Future. Geophys Monogr, Am Geophys Union, 2001, 126: 277-293

23 Hemming S R. Heinrich events: Massive Late Pleistocene detritus layers of the North Atlantic and their global climate imprint. Rev Geophys, 2004, 42, RG1005, doi: 1209/04/2003RG000128

24 Broecker W S, Kennett J P, Flower P, et al. Routing of meltwater from the Laurentide Ice Sheet during the Younger Dryas cold episode. Nature, 1989, 341: 318-321[DOI]

25 Johnson T C, Scholz C A, Talbot M R, et al. Late Pleistocene desiccation of Lake Victoria and rapid evolution of Cichlid fishes. Science, 1996, 273: 1091-1093

26 Stine S. Extreme and persistent drought in California and Patagonia during mediaeval time. Nature, 1994, 369: 546-549 [DOI]

27 Stine S. Late Holocene fluctuations of Mono Lake, eastern California. Palaeogeogr Palaeoclimatol Palaeoecol, 1990, 78: 333381 [DOI]

28 Intergovernmental Panel on Climate Change (IPCC) 2005. World Meteorological Organization (WMO)/United Nations Environmental Programme (UNEP) Intergovernmental Panel on Climate Change (IPCC). In: Rubin E, Meyer L, et al, eds. IPCC Special Report on Carbon Capture and Storage. Available at http://www.ipcc.ch/activity/srccs/index.htm

(2006-01-19 收稿, 2006-03-01 接受) 